\title{
GeoGebra: ferramenta cognitiva para o desenvolvimento do pensamento operatório formal
}

\author{
Jaqueline Molon ${ }^{1}$, Sérgio Roberto Kieling Franco ${ }^{2}$ \\ ${ }^{1}$ Universidade Federal do Rio Grande do Sul (UFRGS) e Instituto Federal de Educação, \\ Ciência e Tecnologia do Rio Grande do Sul (IFRS - Canoas - RS - Brasil - 92412 - \\ 240 Rua Maria Zélia Carneiro de Figueiredo, 870-A \\ ${ }^{2}$ Universidade Federal do Rio Grande do Sul (UFRGS) - Porto Alegre - RS - Brasil \\ jaqueline.molon@canoas.ifrs.edu.br, sergio.franco@ufrgs.br
}

\begin{abstract}
This report aims to discuss the use of GeoGebra as a cognitive tool for knowledge construction at the formal operative level. A report on the tool use in a Flat Geometry subject in a teacher training course is presented. Taking Piaget's Reflexive Abstraction as a theoretical assumption, the proposal contemplated construction, hypothesis raising, conjecture and proof from the observation of invariants identified with the "move" command of the dynamic geometry software. The results indicate that the dynamic geometry use with this orientation can mobilize cognitive skills related to hypothetical-deductive reasoning at the formal operative level.
\end{abstract}

Resumo. $O$ relato tem como objetivo discutir o uso do GeoGebra enquanto ferramenta cognitiva para a construção do conhecimento em nível operatório formal. Apresenta-se um relato de utilização da ferramenta em uma disciplina de geometria plana num curso de formação de professores. Tendo a Abstração Reflexionante de Piaget como pressuposto teórico, a proposta contemplou construção, levantamento de hipóteses, conjectura e prova a partir da observação de invariantes identificados com o comando "mover" do software de geometria dinâmica. Os resultados apontam que a utilização da geometria dinâmica com esse viés pode mobilizar competências cognitivas relacionadas ao raciocinio hipotético-dedutivo em nível operatório formal.

\section{Introdução}

As Tecnologias Digitais da Informação e Comunicação (TDIC) "estão transformando os cenários educacionais tradicionais e, ao mesmo tempo, promovendo o surgimento de outros novos" [Coll e Monereo 2010]. A discussão acerca da incorporação de recursos e ferramentas digitais em práticas de ensino e aprendizagem, por sua vez, encontra-se cada vez mais presente em cursos de formação de professores, tanto incentivada pelas exigências que constam nas diretrizes curriculares de formação docente quanto nos documentos norteadores da educação básica - futuro local de atuação desses licenciandos.

Publicada em 2015 pelo Conselho Nacional de Educação (CNE), a Resolução ${ }^{\circ} 2$ de $1^{\circ}$ de julho, definiu novas diretrizes curriculares nacionais para os cursos de licenciatura, os cursos de formação pedagógica para graduados e os cursos de segunda licenciatura, bem como para a formação continuada. Esse documento destaca que um dos pilares de formação

\footnotetext{
1 Autora possui apoio e fomento do IFRS.
} 
VIII Congresso Brasileiro de Informática na Educação (CBIE 2019)

Anais dos Workshops do VIII Congresso Brasileiro de Informática na Educação (WCBIE 2019)

básica desses profissionais que irão atuar na educação básica pauta-se no "uso competente das Tecnologias de Informação e Comunicação (TIC) para o aprimoramento da prática pedagógica e a ampliação da formação cultural dos(das) professores(as) e estudantes" [Brasil 2015].

No mesmo sentido faz-se referência à Base Nacional Comum Curricular (BNCC) que foi publicada em 2018 e cujo documento final engloba todas as etapas da educação básica (educação infantil, ensino fundamental e ensino médio). A BNCC faz grande consideração ao uso das TDIC em todas as etapas e em todas as áreas da educação básica. De modo especial, na área da matemática, "a BNCC orienta-se pelo pressuposto de que a aprendizagem em Matemática está intrinsecamente relacionada à compreensão, ou seja, à apreensão de significados dos objetos matemáticos, sem deixar de lado suas aplicações" [Brasil 2018]. Nesse sentido destaca que "recursos didáticos como malhas quadriculadas, ábacos, jogos, livros, vídeos, calculadoras, planilhas eletrônicas e softwares de geometria dinâmica têm um papel essencial para a compreensão e utilização das noções matemáticas" [Brasil 2018]. A BNCC contempla de modo específico também a Geometria Dinâmica (GD) que está elencada em nove habilidades específicas da área da matemática a serem desenvolvidas ao longo do ensino fundamental e médio.

Diante desses aspectos, o presente trabalho tem como objetivo ampliar a discussão acerca das potencialidades da utilização do GeoGebra como ferramenta cognitiva para o favorecimento da construção de conhecimentos a partir da geometria dinâmica. Além da conceituação da expressão "ferramentas cognitivas" o texto explora os pressupostos teóricos de Jean Piaget no que tange à construção de novidades, ou seja, à abstração reflexionante [Piaget 1995]. Como pano de fundo para a discussão apresenta-se uma prática didática desenvolvida no contexto da formação de professores ao encontro da integração tecnológica e em favor do desenvolvimento não só do pensamento geométrico - conteúdo específico abordado -, mas também do raciocínio hipotético-dedutivo que é elemento marcante do nível operatório formal do desenvolvimento cognitivo na teoria piagetiana.

\section{Ferramentas Cognitivas e Pensamento Operatório Formal}

O processo de desenvolvimento cognitivo engloba a criação de estruturas novas (cada vez mais complexas) ou a reorganização das existentes para permitir ao sujeito conhecer (assimilar e acomodar) conceitos cada vez mais complexos [Piaget 1995]. O ponto fundamental para a construção de conceitos pelo sujeito é a sua capacidade de construir estruturas que não estão dadas nos objetos por si só e nem no próprio sujeito, mas na coordenação das ações desse sujeito com o objeto a ser conhecido, o que Piaget chamou de Abstração Reflexionante [Piaget 1995]. Essa abstração "se apoia sobre as formas e sobre todas as atividades cognitivas do sujeito (esquemas, coordenações de ações, operações, estruturas, etc.) que delas retira certos caracteres e os utiliza para outras finalidades" [Becker 2013].

A construção de um conhecimento novo promove modificações no comportamento do sujeito. De acordo com Franco (1997) "todo conhecimento novo gera novidade, não só pelo conteúdo adquirido, mas principalmente pela formalização que o sujeito constrói sobre este conteúdo". O pensamento dedutivo é alcançado quando o sujeito consegue pensar independente do conteúdo. Através de abstrações reflexionantes o sujeito consegue tirar conclusões que independem da observação de elementos reais, a partir das formas ou estruturas de raciocínio, as quais permitem estabelecer relações entre os conteúdos. A 
construção das estruturas operatório-formais torna o sujeito capaz de realizar raciocínio hipotético-dedutivo, de conseguir operar sobre os possíveis (analisar possibilidades), de construir o pensamento probabilístico, combinatório e as proporções, além de levantar hipóteses, elaborar conjecturas e realizar demonstrações.

O conceito de ferramentas cognitivas aproxima as TDIC do contexto de promoção ou desenvolvimento de habilidades ou competências cognitivas. Essas ferramentas podem ser consideradas como recurso para a transição do pensamento operatório concreto ao operatório formal na perspectiva de construção do conhecimento. De acordo com Varela, Barbosa e Farias (2014) ferramentas cognitivas são "todas as tecnologias ou aplicações que buscam facilitar a aprendizagem significativa e o pensamento crítico, tendo como foco a reflexão, a construção do conhecimento, a colaboração, a conversação e a articulação". Na mesma direção Korres (2019) citada por Silva (2019) defende que as ferramentas cognitivas são recursos computacionais que apoiam a construção do conhecimento dos estudantes, possibilitando etapas de coleta, organização e análise de dados de determinado problema, elaboração de conjecturas e conceitos e a chegada a uma solução ou conclusão.

As TDIC podem se tornar grandes aliadas dos processos de ensino e de aprendizagem na medida em que sua incorporação tende a promover espaços de experimentação, tentativa e validação de conceitos ou hipóteses. O GeoGebra é uma tecnologia que pode ser utilizada em diferentes dispositivos (computadores, tablets, smartphones, etc.) sendo bastante intuitivo e, por isso, a sua incorporação em atividades no ambiente escolar é facilitada. O GeoGebra não é apenas uma ferramenta de geometria dinâmica, mas tal funcionalidade é um dos grandes diferenciais desse recurso em termos de promoção de aprendizagem. O dinamismo das construções, ou seja, a possibilidade de modificá-las a partir de recursos disponíveis na ferramenta abre espaço para a criatividade, a observação, o levantamento e a testagem de hipóteses aspectos que são essenciais para a argumentação e demonstração em matemática. Essas habilidades são fundamentais para o desenvolvimento cognitivo dos estudantes, especialmente em termos de evolução do pensamento operatório concreto ao operatório formal e, justamente, são esses os elementos que possibilitam considerar o GeoGebra como uma ferramenta cognitiva.

\section{Relato de Experiência: metodologia, desenvolvimento e discussão}

Este relato refere-se a análise de uma atividade de construção, conjectura e demonstração matemática proposta a uma turma de estudantes da disciplina de Geometria I incluída no $3^{\circ}$ semestre da matriz curricular de um curso de Licenciatura em Matemática de uma instituição federal de educação da região metropolitana de Porto Alegre.

A coleta dos dados ocorreu no primeiro semestre de 2018 e contou com a participação de 15 alunos. Os dados foram coletados a partir dos arquivos construídos por cada estudante no software GeoGebra e submetidos no ambiente virtual de aprendizagem Moodle. A descrição da atividade proposta encontra-se no quadro 1.

Percebe-se que a utilização do GeoGebra para a realização dessa atividade tem um objetivo bastante específico: envolver os alunos em uma atividade de construção, elaboração de conjecturas e investigação pautada sob o dinamismo da construção possibilitado pela utilização da ferramenta. Observa-se que se a mesma atividade fosse realizada apenas com lápis e papel, a construção ficaria restrita a apenas uma representação (ou limitada a algumas construções) de modo que a elaboração da generalização - todo 
VIII Congresso Brasileiro de Informática na Educação (CBIE 2019)

Anais dos Workshops do VIII Congresso Brasileiro de Informática na Educação (WCBIE 2019)

quadrilátero $M N P Q$ construído com vértices nos pontos médios de um quadrilátero qualquer é um paralelogramo - poderia ser dificultada.

\section{Quadro 1. Orientações fornecidas aos estudantes acerca da atividade de construção}

a) Construa, no GeoGebra, um quadrilátero $\mathrm{ABCD}$. Localize os pontos: $\mathrm{M} \mathrm{o}$ ponto médio de $\overline{A B}$, N o ponto médio de $\overline{B C}$, $\mathrm{P}$ o ponto médio de $\overline{C D}$ e $\mathrm{Q}$ o ponto médio de $\overline{A D}$. Construa o quadrilátero MNPQ.

b) Utilize o comando "mover" para modificar o quadrilátero $A B C D$ e observe o quadrilátero construído a partir dos respectivos pontos médios de $\mathrm{ABCD}$. Que característica invariante possui o quadrilátero MNPQ? Utilize o recurso texto para registrar sua conjectura e a argumentação matemática para validar sua hipótese.

c) Submeta o arquivo (.ggb) na tarefa destinada a essa atividade disponível no ambiente virtual (Moodle) da disciplina.

Além disso, a exploração das propriedades que possibilitam a prova matemática dessa conjectura também é favorecida por intermédio do GeoGebra enquanto ferramenta cognitiva. A representação geométrica da situação, em contexto de construção dinâmica, representa um importante apoio para o desenvolvimento da capacidade de elaboração de deduções a partir da observação de propriedades invariantes diante do movimento.

Deduzir uma propriedade significa estabelecer uma cadeia lógica de raciocínios conectando propriedades do enunciado tomados como pressupostos (hipóteses) às propriedades ditas decorrentes (teses). Esta cadeia de raciocínios que denominamos de argumentação lógica e dedutiva. $\mathrm{O}$ desenho entra aqui como materialização da configuração geométrica, guardando as relações a partir das quais decorrem as propriedades [Gravina 1996].

Esse processo colabora para a realização de abstrações reflexionantes e, consequentemente, para a projeção de um conhecimento representado de forma concreta para a elaboração de um conceito formal, uma generalização, cuja demonstração exige a compreensão dos elementos envolvidos e das relações entre eles em termos de estruturação lógica, ou seja, de concatenação argumentativa que implica na utilização de pensamento operatório formal (raciocínio hipotético-dedutivo). A abstração reflexionante engloba dois processos: no primeiro o sujeito projeta a um novo patamar um conhecimento construído em um patamar inferior (processo de reflexão) e, posteriormente ele reorganizar suas estruturas cognitivas considerando essa nova construção (processo de reflexionamento) [Piaget 1995].

Esse processo de passagem da representação geométrica à argumentação matemática pode ser observado na Figura 1, construção realizada pelo estudante 10 (E10). 
VIII Congresso Brasileiro de Informática na Educação (CBIE 2019)

Anais dos Workshops do VIII Congresso Brasileiro de Informática na Educação (WCBIE 2019)

Ao construímos um quadrilätero qualquer $\mathrm{ABCD}$.

Sabemos que os ponto médio $M, N, P$ e $Q$ são respectivos pontos médios dos segmentos $A B, B C, C D$ e $A D$

Ao traçarmos segmentos de MN, NP, $P Q$ e também QM queremos provar que MNPQ é um paralelogramo (lados opostos paralelos). Ao traçarmos um diagonal de $\mathrm{BD}$ obtemos dois triângulos: $\mathrm{ABD}$ e $\mathrm{BCD}$.

Considerando o triângulo $A B D$, sabemos que $M$ e $Q$ são pontos médios dos respectivos segmentos $A B$ e $A D$, sendo assim o segmento $M Q$ é base média relativa a base $\mathrm{BD}$ do triângulo, ou seja, $\mathrm{MQ}$ também é paralela ao segmento $\mathrm{BD}$ ( também base do triângulo $\mathrm{ABD}$ ). Considerando o triângulo $B C D$. Sabemos que $N$ e $Q$ são pontos médios dos respectivos segmentos $B C$ e $C D$, sendo assim o segmento $N P$ é base média relativa a base $\mathrm{BD}$ do triângulo $\mathrm{BCD}$.

Ao traçarmos a outra diagonal $\mathrm{AC}$, obtemos dois triângulos: $\mathrm{ABC}$ e $\mathrm{ADC}$

Considerando o triângulo $A B C$, sabemos que $M$ e N são pontos médios dos respectivos segmentos $A B$ e $B C$, sendo assim o segmento $M N$ é base média relativa a base $\mathrm{AC}$ do triângulo $\mathrm{ABC}$, ou seja, $\mathrm{MN}$ também é paralela a $\mathrm{AC}$ (base do triângulo).

Considerando o triângulo $A D C$, sabemos que $Q$ e $P$ são pontos médios dos respectivos segmentos $A D$ e $D C$, sendo assim o segmento $Q P$

é base média relativa a base do triângulo (AC), sendo também, paralela a AC

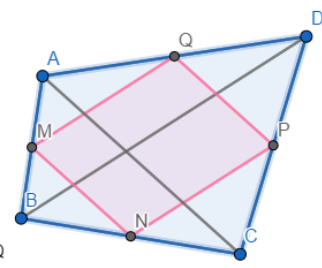

Podemos concluir que

${ }^{*} \mathrm{MN} / / \mathrm{AC}, \mathrm{QP} / / \mathrm{AC}$, sendo assim $\mathrm{MN} / / \mathrm{QP}$.

${ }^{*} \mathrm{MQ} / / \mathrm{BD}$, e que $\mathrm{NP} / / \mathrm{BD}$, sendo assim, MQ//NP.

O que concluimos que MNQP é um paralelogramo, pois MN// QP e MQ// NP. (lados opostos paralelos)

Figura 1. Construção elaborada por E10

Destaca-se que dos 15 estudantes que realizaram a atividade apenas um deles (E1) recorreu a recursos de medição de segmentos e apresentou como verificação da validade da conjectura, apenas alguns casos específicos (os que foram obtidos e medidos por ele!) (Figura 2).
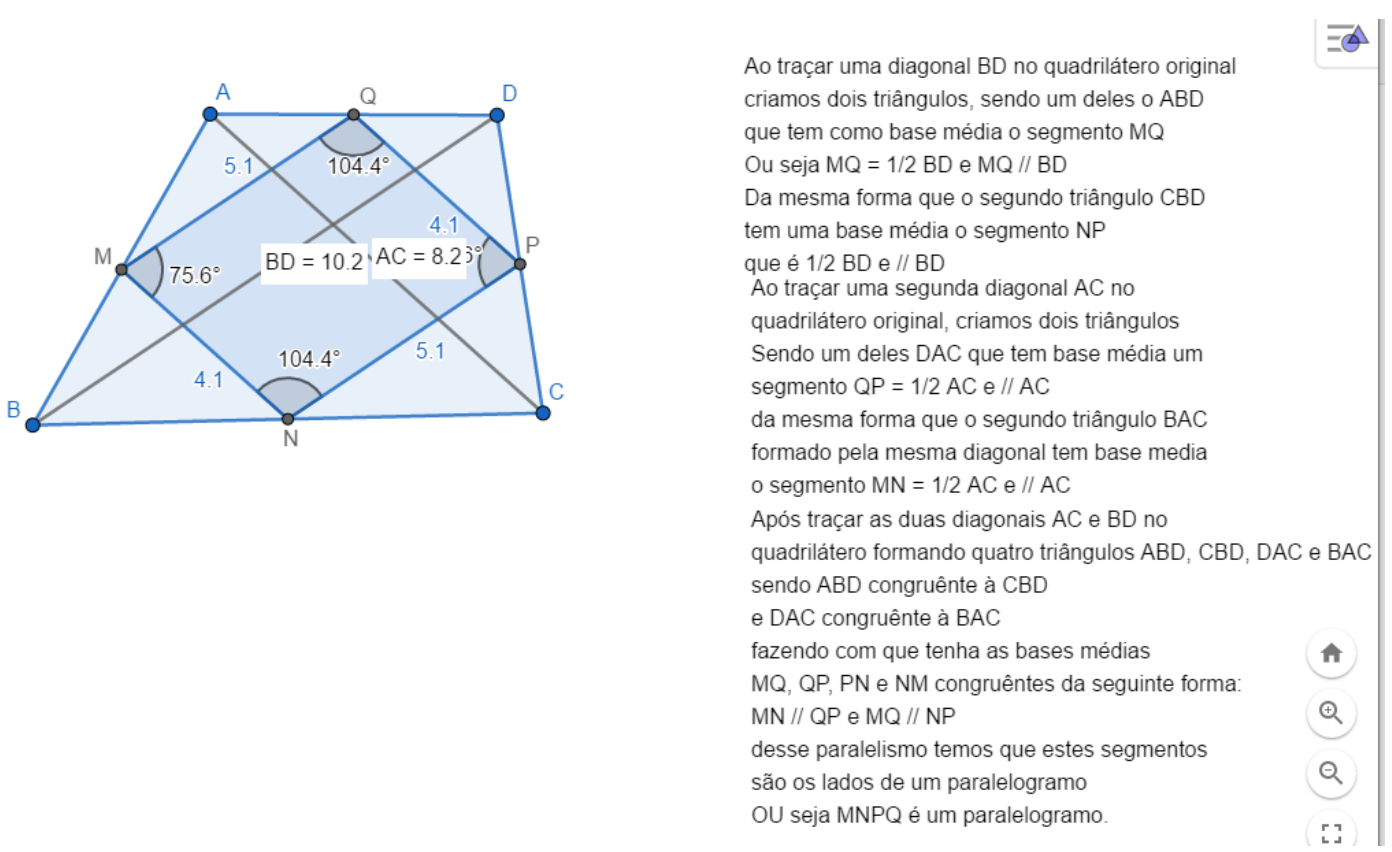

Figura 2. Construção elaborada por E1

No entanto, o estudante elaborou a justificativa generalizando suas conclusões, o que demonstra que ele está em processo de construção e elaboração do raciocínio hipotético-dedutivo, porém em etapa que exige ainda o apoio de elementos concretos (ou seja, da medição dos segmentos e ângulos). Analisando o desempenho desse estudante nas atividades que exploraram as propriedades dos quadriláteros notáveis estudadas antes da realização dessa atividade, observou-se evolução em sua capacidade argumentativa. 
VIII Congresso Brasileiro de Informática na Educação (CBIE 2019)

Anais dos Workshops do VIII Congresso Brasileiro de Informática na Educação (WCBIE 2019)

\section{Considerações Finais}

Esse artigo trouxe para discussão o GeoGebra como ferramenta cognitiva e refletiu sobre a utilização da geometria dinâmica enquanto recurso para a construção do raciocínio hipotético-dedutivo, que se dá em nível operatório formal de acordo com a teoria piagetiana.

$O$ relato de experiência apresentado evidencia que atividades que englobam construção, levantamento de hipóteses, conjectura e prova a partir da observação de invariantes são potencializadas com a utilização de ambientes de geometria dinâmica. Tais recursos disponíveis nesses espaços possibilitam a construção/reprodução gráfica dos objetos e podem conduzir os estudantes à abstração de conceitos geométricos envolvidos durante a realização de atividades pautadas na investigação e busca por regularidades, generalizações e demonstração. O GeoGebra enquanto ferramenta cognitiva favorece o "pensar" e possibilita a experimentação e o teste de hipóteses.

Considera-se fundamental, por sua vez, que atividades com esse viés investigativo sejam proporcionadas aos estudantes para que eles vivenciam, enquanto alunos, tanto as dificuldades quanto as potencialidades do uso das TDIC nos processos de ensino e de aprendizagem. A formação inicial dos professores deve oportunizar reflexões também acerca da interpretação do erro como processo e, a utilização de uma ferramenta digital, valoriza os processos de construção e reconstrução, validando as tentativas dos estudantes, o que seria muito oneroso se tal proposta fosse executada sem auxílio tecnológico.

\section{Referências}

Becker, F. (2013) "Sujeito do Conhecimento e Ensino de Matemática", http://www2.marilia.unesp.br/revistas/index.php/scheme/article/view/3222/2517, September.

Brasil. (2015) "Resolução $\mathrm{n}^{0} 2$, de 01 de julho de 2015", http://portal.mec.gov.br/index.php?option=com_docman\&view=download\&alias=17719 -res-cne-cp-002-03072015\&category_slug=julho-2015-pdf\&Itemid=30192, September.

Brasil. (2018) "Base Nacional Comum Curricular BNCC". http://basenacionalcomum.mec.gov.br/images/BNCC_EI_EF_110518_versaof inal_site.pdf , September.

Coll, C. and Monereo, C. (2010), Psicologia da educação virtual - Aprender e Ensinar com as Tecnologias da Informação e da Comunicação, Artmed, Porto Alegre.

Dolce, O. and Pompeo, J. N. (2013), Fundamentos da Matemática Elementar, Atual, São Paulo.

Franco, S.R.K. (1997) "Piaget e a Dialética", In Revisitando Piaget, pages 9-20. Mediação.

Gravina, M. A. (1996). Geometria Dinâmica: Uma Nova Abordagem para o Aprendizado da Geometria. In Anais do VII Simpósio Brasileiro de Informática na Educacão, pages $1-13$.

Piaget, J. (1995), Abstração reflexionante: relações lógico-aritméticas e ordem das relações espaciais, Artes Médicas. 
VIII Congresso Brasileiro de Informática na Educação (CBIE 2019)

Anais dos Workshops do VIII Congresso Brasileiro de Informática na Educação (WCBIE 2019)

Silva, Patrícia Fernanda da. (2019) "O uso das Tecnologias Digitais como Ferramentas Cognitivas", https://seer.ufrgs.br/renote/article/view/96588, September.

Varela, A.V; Barbosa, M.L.A and Farias, M.G.G. (2014) "Ferramentas cognitivas, ambientes modificadores, medição e construção do conhecimento: potencializando a cognição do sujeito social na perspectiva do aprender", http://revista.ibict.br/ciinf/article/view/1404, September. 